\title{
La competencia matemática de la representación, desde la linealidad ${ }^{1}$
}

\section{Mathematical competence of representation, from the linearity}

\section{A competência matemática da representação, a partir da linearidade}

Recibido: mayo 2013

Aceptado: agosto 2013
José Arley Londoño $\mathrm{A}^{2}$.

Eliécer Aldana Bermúdez ${ }^{3}$

\section{Resumen}

Este estudio reporta los resultados de una investigación en curso, donde se busca el desarrollo de competencias matemáticas en el pensamiento variacional desde el concepto de función lineal. La investigación tiene como base la teoría de "Las Situaciones Didácticas". Para ello se ha utilizado, como metodología una ingeniería didáctica a un grupo de estudiantes de grado noveno, a los cuales se les aplicó un cuestionario y una entrevista. A partir del análisis y de los resultados se muestran algunas dificultades que ellos ponen de manifiesto en la resolución de problemas que requieren de la modelación de la función lineal.

Palabras clave: Función lineal; Ingeniería Didáctica; Representación; Situaciones Didácticas; Competencias; Matemáticas Escolares; Álgebra; Funciones.

\begin{abstract}
This study reports the results of an ongoing investigation, which seeks to develop math skills in thinking from concept variational linear function. The research is based on the theory of "The Didactic Situations". This has been used as teaching engineering methodology to a group of ninth grade students, to which was applied a questionnaire and an interview. From the analysis and the results are some difficulties they are reflected in the resolution of problems which require the modeling of the linear function.
\end{abstract}

Keywords: linear function, Engineering Teaching; Representation; Didactic Situations, Skills, School Mathematics, Algebra, Functions.

\section{Resumo}

Este estudo apresenta os resultados de uma investigação em curso, que visa a desenvolver habilidades matemáticas no pensamento do conceito de função linear variacional. A pesquisa é baseada na teoria das "Situações Didáticas". Este foi usado como ensinar a metodologia de engenharia de um grupo de

Artíulo de Investigación

2 Institución Educativa Robledo. Contacto: arleymate@yahoo.es

3 Universidad del Quindío. Contacto: eliecerab@uniquindio.edu.co 
estudantes nono grau, ao qual foi aplicado um questionário e entrevista. A partir da análise e os resultados são algumas dificuldades que se refletem na resolução de problemas que exigem a modelagem da função linear.

Palavras-chave: função linear, ensino de engenharia; Representação; Situações Didáticas, Skills, Matemática Escolar, Álgebra, Funções.

\section{Presentación del problema}

Los resultados de investigaciones acerca del concepto reflejan algunas dificultades de comprensión. Algunas de estas dificultades apuntan al mismo sistema escolar, a los docentes, a las familias, a los estudiantes y a la falta de competencias que estos presentan al enfrentarse a desafíos que implican la utilización de herramientas matemáticas. Al respecto, Santos y Alvarado (2000) reportan la falta de aprendizaje del concepto debido al énfasis que se hace en la enseñanza de procedimientos algorítmicos, y la carencia de una enseñanza basada en la resolución de problemas. Duval (1995) plantea que una causa importante del fracaso escolar está ligada a la conversión entre diferentes registros de representación: los estudiantes saben, en general, trabajar aisladamente con cada una de ellos, sin embargo, no tienen la capacidad de decidir si conviene o no cambiar de registro según la tarea que se les presente. Por tanto lo que se espera es ¿Cómo generar en el estudiante de grado noveno el desarrollo de la competencia matemática de la representación, en torno al concepto de función lineal?

\section{Marco de referencia conceptual.}

El foco de atención lo constituye la competencia de la representación en el pensamiento variacional, desde el concepto función lineal, porque requiere de la formación de conceptos apropiados, cuando el sujeto tiene que resolver tareas en contextos específicos que requieren del uso de los elementos matemáticos necesarios y coordinar diferentes registros de representación. Para desarrollar esta investigación nos apoyamos en la Teoría de las Situaciones Didácticas de Brousseau, (1997) y de la Teoría de la Transposición Didáctica Chevallard (1991). Una situación didáctica es un conjunto de relaciones establecidas entre un estudiante o un grupo de estudiantes. La primera fase de una situación didáctica, es la a-didáctica, en la cual el profesor no interviene dejando que el estudiante viva esta situación como investigador de un problema matemático Margolinas, (1993). La segunda fase es la didáctica propiamente, en este nivel se tiene en cuenta la modelización algunas limitaciones del modelo a-didáctico, considerando al estudiante como un sujeto institucional cuyos procesos de adaptación están condicionados por la tarea matemática y las interacciones posibles con el medio, la noción central en este nivel es la de "contrato didáctico" que designa las expectativas implícitas que tienen relación con el otro, con el estudiante y el docente, frente al saber matemático. Es aquí, donde el investigador mediante secuencias didácticas de enseñanza apoyadas en diseños de tareas específicas permite generar en el estudiante el desarrollo de la competencia de la representación en torno a la función lineal.

\section{Metodología}

La metodología está fundamentada en la ingeniería didáctica de Chevallar (1997), es una investigación de tipo cualitativo, que según Merriam (1998, p: 6), consiste en: a) entender el fenómeno de interés desde las perspectivas de los participantes y no del investigador, b) el investigador es el instrumento primario para la colección de datos y el análisis, c) esta metodología se refiere al estudio de casos, d) emplea la estrategia de investigación inductiva, e) se enfoca en los procesos y entendimientos. Este es un estudio de caso con estudiantes de grado noveno y se hizo en diferentes fases: diseño, elaboración y aplicación de unas situaciones a-didácticas; análisis a-priori de las situaciones a-didácticas, modificación y elaboración de las situaciones didácticas; aplicación de algunas situaciones didácticas, análisis a posteriori, realización de entrevistas, triangulación de la información y conclusiones. 


\section{Análisis de datos}

En primer lugar se analizan los instrumentos aplicados durante las situaciones a-didácticas desde los aspectos epistemológicos que tiene que ver con los elementos constitutivos del concepto matemático, lo didáctico desde lo que hace el profesor y lo cognitivo sobre la forma cómo comprenden los estudiantes en los cuestionarios y entrevistas. Esto es lo que hace un estudiante durante la fase a-didáctica:

Figura 1: Tarea 4 del cuestionario 1 a-didáctico.

\section{4) La ley de Hooke (más conocido como la ley del resorte) establece la relación que existe entre la fuerza. F aplicada a un resorte y el estiramiento $L$. producido en éste. \\ a) Completar la siguiente tabla.}

\begin{tabular}{|r|r|r|r|r|r|}
\hline F(dyn) & 10 & 15 & 20 & 45 & 50 \\
\hline $\mathrm{L}(\mathrm{cm})$ & 2 & & & 9 & \\
\hline
\end{tabular}

b) Representa gráficamente estos datos sobre un sistema de ejes cartesianos.

c) A partir del gráfico, ¿puedes obtener las fuerzas que corresponden a un estiramiento de $5 \mathrm{~cm}$ ¿y $3.3 \mathrm{~cm}$ ? ¿y $8 \mathrm{~cm}$ ?

d) ¿Que estiramiento corresponde a una fuerza de 30 dyn? ¿y 5 dyn? ¿55 dyn?

e) ¿Cómo enunciar la ley de Hooke?

Fuente: Elaboración propia

En la respuesta se identifica las herramientas utilizadas por el estudiante, para establecer la solución a las preguntas planteadas con base en la gráfica de la recta obtenida.

Figura 2: Respuesta planteada por el estudiante E5

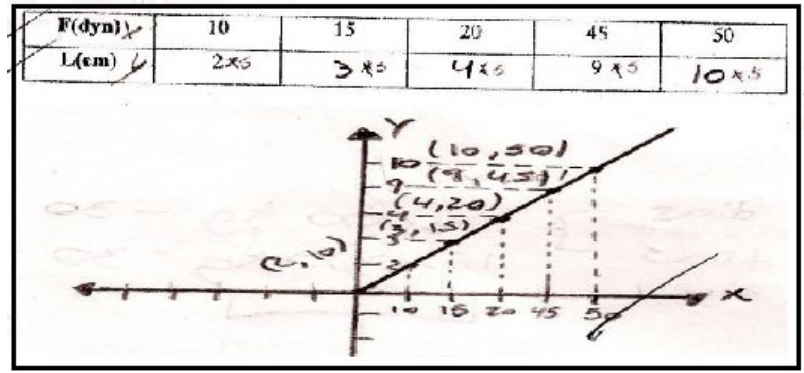

Fuente: Elaboración propia

Hay un desempeño adecuado por el estudiante, muestra que hay comprensión y reconocimiento de la gráfica, identifica características, relacionadas con las magnitudes y proporciones. Además no utiliza argumentos matemáticos que ayuden a interpretar los elementos que caracterizan la función lineal. Durante la entrevista esto es lo que ponen en evidencia:
P: ¿Cómo has usado las condiciones del enunciado de la tarea para pensar en ¿las soluciones?

R.E: Como la mayoría de estos ejercicios del análisis preliminar era llenar tablas entonces comencé a observar cada una de ella y mire que algunas eran fáciles solo con multiplicaciones de algunos valores.

$\mathrm{P}:$ ¿Qué relación hay entre las variables presentadas en los puntos anteriores?

R.E: La tabla representada en el ejercicio 4 del análisis preliminar la variable independiente es La fuerza mientras que la variable dependiente es el estiramiento del resorte.

$\mathrm{P}:$ ¿Por qué usted representó por medio de una función lineal los puntos anteriores? Justifique la respuesta

R.E: Por que como algunos enunciados decía que planteara los ejercicios en un sistema cartesiano tomando los valores que había en la tabla comencé a formar parejas ordenadas y después los uní formando una línea recta.

Lo que busca por medio de la representación es reconocer la interpretación rápida y precisa, lo cual indica que hay un reconocimiento de los elementos gráficos que configuran la función lineal; sin embargo en esta tarea se pretende que el sujeto mediante un proceso lógico transforme una representación gráfica hasta lograr una expresión algebraica. 
Figura 3: Tarea 1 del cuestionario 1 didáctico.

1.Iremos de vacaciones a la playa y nos espera un viaje de $500 \mathrm{Km}$. Sabemos que el auto consume 2 galones de gasolina cada $100 \mathrm{Km}$ ¿Cuántos galones necesitamos para el viaje?

Respuesta detallada.

Fuente: Elaboración propia

Esto es lo que hace el estudiante durante la resolución de la tarea en el cuestionario.

Figura 4: Respuesta planteada por el estudiante E7

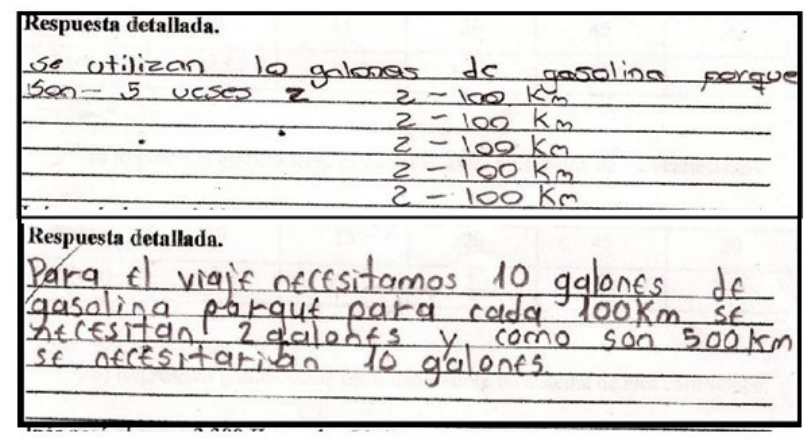

Fuente: Elaboración propia

Podemos ver la evolución de algunos objetos matemáticos importantes entre las competencias trabajadas como la de resolver problemas a través de las magnitudes establecidas en el análisis. Como se pone en evidencia el estudiante establece algunos argumentos matemáticos que justifican los procesos analíticos aplicados.

\section{Conclusiones}

Los estudiantes en la dimensión a-didáctica no reconocen los elementos que configuran la función lineal y no relacionan los modos de representación gráfico y algebraico, sin embargo en un segundo momento en la fase didáctica, dónde existe una mediación del profesor investigador, se nota un cambio en el aprendizaje, donde se aprecia que los sujetos son capaces de reconocer, relacionar y transformar los datos de un modo de representación a otro, siempre apoyados en la construcción de la función lineal, sin embargo el registro privilegiado para esta conversión es el registro gráfico, por las numerosas unidades significantes que posee y la correspondencia de estas con el registro verbal, entre ellas las magnitudes, los valores que toma cada una de las variables, las escalas, la pendiente de covariación, la continuidad de la función, además es una representación claramente reconocida para este objeto matemático. Las actividades planteadas mediante secuencias didácticas de enseñanza permitieron el desarrollo de la competencia de representación como el camino para resolver las situaciones porque permite hacer una comparación no solo aritmética sino visual, generando seguridad en el momento de tomar decisiones.

\section{Referencias}

Artigue, M. (1998 ). Ingeniería didactica. En Artigue, M, Douay, R, Moreno, L,Gomez, P. (Eds). Ingeniería didáctica en educación matemática. Colombia : Una empresa docente .

Brousseau, G. (1997). Theory of Didactical Situations in Mathematics. Kluwer Academic Publishers.

Caicedo, S.J. Pensamiento variacional de estudiantes de grado noveno en el proceso de resolución de situaciones problema que se pueden modelar con una función cuadrática. Universidad del tolima. Tesis doctoral no publicada. 2013.

Chevallard, Y. (1991). La transposición didáctica: del saber sabio al saber enseñado. Buenos Aires.: Aique. 